\title{
On a diophantine equation in two unknowns
}

\author{
By Bengt Persson
}

\section{$\S 1$.}

The purpose of this paper is to examine the solvability in integers $x$ and $y$ of the equation

$$
x^{2}+x+\frac{1}{4}(D+1)=y^{q}
$$

where $D$ is a positive integer $\equiv 3(\bmod .4)$ and $q$ denotes an odd prime.

The special case $D=3$ has already been treated by T. NAGELL, who showed that the equation ${ }^{1}$

$$
x^{2}+x+1=y^{n}
$$

is impossible in integers $x$ and $y$, when $y \neq \pm 1$, for all whole exponents $n(>2)$ not being a power of 3 .

W. LJUNGGReN completed this result by proving that the equation ${ }^{2}$

$$
x^{2}+x+1=y^{3}
$$

has the only solutions $y=1$ and $y=7$. Thus it is sufficient in (1) to take $D \geqq 7$. We furthermore suppose that $D$ has no squared factor $>1$.

According to a theorem of AxEL THUE the equation (1) has only a finite number of solutions in integers $x$, and $y$, when $D$ and $q$ are given. ${ }^{3}$

\section{$\S 2$.}

If we put $\varrho=\frac{1}{2}(-1+\sqrt{-D})$ and $\varrho^{\prime}=\frac{1}{2}(-1-\sqrt{-\bar{D}})$, the equation (1) can be written

$$
(x-\varrho)\left(x-\varrho^{\prime}\right)=y^{q} \text {. }
$$

$\varrho$ and $\varrho^{\prime}$ are conjugate integers in the quadratic field $K(\sqrt{-D})$. The numbers $1, \varrho$ form a basis of the field.

The two principal ideals

$$
(x-\varrho) \text { and }\left(x-\varrho^{\prime}\right)
$$

are relatively prime. To show it we denote by $j$ their highest common divisor. The number $2 x+1=2 x-\left(\varrho+\varrho^{\prime}\right)$ is contained in $\dot{j}$ and also the number $D=-\left(\varrho-\varrho^{\prime}\right)^{2}$. If we write the equation (1) in the form 
B. PERSSON, On a diophantine equation in two unknowns

$$
(2 x+1)^{2}+D=4 y^{q}
$$

we see that the numbers $2 x+1$ and $D$ are relatively prime since $D$ has no squared factor $>1$. Hence we have $i=(1)$. Therefore we get from (1') the ideal equation

$$
(x-\varrho)=\mathfrak{a}^{q}
$$

where $\mathfrak{a}$ is an ideal. Let us for the present suppose that $\mathfrak{a}$ is a principal ideal. Then (2) can be written

$$
x-\varrho=\varepsilon(a+b \varrho)^{q}
$$

where $a$ and $b$ are relatively prime integers and $\varepsilon$ is a unit in $K(\stackrel{\bullet}{-\bar{D}})$. $D$ being $>3$, the only units are \pm 1 . Thus the unit $\varepsilon$ is a $q$-th power, and we can replace $\varepsilon$ by 1 .

Hence we get from $\left(2^{\prime}\right)$

$$
x-\varrho=(a+b \varrho)^{q}=\left(a-\frac{b}{2}+\frac{b}{2} \sqrt{-D}\right)^{q}=\frac{(c+b \sqrt{-D})^{q}}{2^{q}}
$$

with

$$
c=2 a-b
$$

From (3) we get

or developed

$$
2^{q} \sqrt{-D}=(c-b \sqrt{-D})^{q}-(c+b \sqrt{-D})^{q}
$$

$$
2^{q-1}=-\sum_{r=0}^{\underline{z}(q-1)}\left(\begin{array}{c}
q \\
2 r+1
\end{array}\right) c^{q-2 r-1} b^{2 r+1}(-D)^{r} .
$$

From (4) we get $b= \pm 2^{m}$. Here $m=0$ is the only possibility, for otherwise $c$ would be even too by (3) and the right member of (4) would be divisible by $2^{q}$. Hence $b= \pm 1$.

From (4) we get modulo $q$

Hence

$$
1 \equiv-b(-D)^{\frac{3}{2}(q-1)} \quad(\bmod , q) .
$$

$$
b=-\left(\frac{-D}{q}\right)
$$

Then the equation (4) is transformed into

$$
2^{q-1}\left(\frac{-D}{q}\right)=\sum_{r=0}^{\frac{1}{2}(q-1)}\left(\begin{array}{c}
q \\
2 r+1
\end{array}\right) c^{q-2 r-1}(-D)^{r},
$$

which is an algebraic equation in $c^{2}$ of degree $\frac{1}{2}(q-1)$ and with integral coefficients. To every integral solution $\pm c$ of the equation (5) corresponds one integral solution $y$ of the equation (1) given by

$$
y=N(\mathfrak{a})=\frac{1}{4}\left(D+c^{2}\right) .
$$


In this way we can have at most $\frac{1}{2}(q-1)$ solutions $y$ of (1), when $D$ and $q$ are given.

The right member of (5) is a binary form of degree $n=\frac{1}{2}(q-1)$ in $c^{2}$ and $D$. This form is irreducible; to see it we regard the polynomial in $z$

$$
f(z)=\sum_{r=0}^{\frac{1}{2}(q-1)}\left(\begin{array}{c}
q \\
2 r+1
\end{array}\right) z^{r}=\sum_{r=0}^{n} a_{r} z^{r}
$$

which has the following properties: $a_{n} \neq 0(\bmod . q) ; a_{i} \equiv 0(\bmod , q)$ for all $i<n ; a_{0} \neq 0\left(\bmod . q^{2}\right)$.

Hence $f(z)$ is irreducible according to the theorem of Eisenstein. Using the wellknown theorem of AXEL THUE ${ }^{4}$ on the corresponding form

$$
f(x, y)=y^{n} f\left(\frac{x}{y}\right)
$$

we see that the equation (5) has only a finite number of solutions in integers $c^{2}$ and $D$, when $q$ is given and $\geqq 7$.

\section{$\S 3$.}

Let us denote by $h(\sqrt{-\bar{D}})$ the number of ideal classes in the field $K(\sqrt{-\bar{D}})$. We shall prove the following proposition:

Theorem 1. If $D$ is a positive integer $\equiv 3$ (mod. 4) having no squared factor $>1$ and if $h(\sqrt{-D})$ is not divisible by the prime $q$, the equation

$$
x^{2}+x+\frac{1}{4}(D+1)=y^{q}
$$

is solvable in integers $x$ and $y$ only for a finite number of integers $D$ for a given $q \geqq 7$. The equation has at most $\frac{1}{2}(q-1)$ solutions $y$, when $D$ and $q$ are given.

In consequence of the results in the preceding paragraph the theorem is proved, when we can prove that in (2) the ideal $\mathfrak{a}$ is a principal ideal if $h=h(\sqrt{-D})$ is not divisible by $q$.

For if $h \neq 0(\bmod . q)$ there are two integers $f$ and $g$ so that

$$
f q-g h=1 \text {. }
$$

Hence we get from (2) the equivalence

$$
\mathfrak{a} \sim \mathfrak{a}^{f \mathfrak{q}} \sim(1)
$$

From the relation (6) we see that if the equation (1) has a solution $y<\frac{1}{4}(D+1)$, we must have $h(V-D) \equiv 0$ (mod. $q$ ). So we get the following result: 


\section{B. PERSSON, On a diophantine equation in two unknowns}

Theorem 2. Let $x$ and $y$ be any integers so that

and so that the number

$$
y^{q}-y>x^{2}+x
$$

$$
D=4 y^{q}-(2 x+1)^{2}
$$

has no squared factor $>1$. Then the number $h(\sqrt{-D})$ is divisible by the odd prime $q$.

\section{Numerical examples}

1. If $q=3, y=2$ and $x=1$ we get $D=4 \cdot 2^{3}-3^{2}=23$ with $2^{3}-2>1^{2}+1$. Hence $h(\sqrt{-23}) \equiv 0(\bmod .3)$.

2. If $q=5, y=2$ and $x=4$ we get $D=4 \cdot 2^{5}-9^{2}=47$ with $2^{5}-2>4^{2}+4$. Hence $h(\sqrt{-47}) \equiv 0(\bmod .5)$.

3. If $q=7, y=2$ and $x=10$ we get $D=4 \cdot 2^{7}-21^{2}=71$ with $2^{7}-2>10^{2}+10$. Hence $h(\sqrt{-71}) \equiv 0(\bmod .7)$.

4. If $q=11, y=2$ and $x=44$ we get $D=4 \cdot 2^{11}-89^{2}=271$ with $2^{11}-2>44^{2}+44$. Hence $h(\sqrt{-271}) \equiv 0(\bmod .11)$.

\section{$\S 4$.}

We shall determine an upper limit for the solutions $y$ of the equation (1), when the number $h(\sqrt{-\bar{D}})$ is not divisible by $q$. As was shown in the preceding paragraph the ideal $\mathfrak{a}$ in (2) is then a principal ideal.

Let us write $\left(3^{\prime}\right)$ as a product. From $\left(3^{\prime}\right)$ we get

$$
\begin{gathered}
2^{q} \sqrt{-D}=\alpha^{q}-\alpha^{q} \quad \text { with } a=c-b \sqrt{-D} . \\
2^{q} \sqrt{-D}=-2 b V \overline{-D} \prod_{r=1}^{q-1}\left(\alpha-\alpha^{\prime} e^{\frac{2 \pi i}{q} r}\right) \\
2^{q-1}\left(\frac{-D}{q}\right)=\prod_{r=1}^{q-1} e^{\frac{\pi i}{q} r}\left(\alpha e^{-\frac{\pi i}{q} r}-\alpha^{\prime} e^{\frac{\pi i}{q} r}\right)= \\
=(-1)^{\frac{z}{(q-1)}} \prod_{r=1}^{q-1} 2\left(-i c \sin _{q}^{\frac{\pi}{q} r}-b V \overline{-D} \cos \frac{\pi}{q} r\right)= \\
==2^{q-1} \prod_{r=1}^{q-1}\left(c \sin \frac{\pi}{q} r+b \sqrt{D} \cos \frac{\pi}{q} r\right) .
\end{gathered}
$$

Hence

$$
\left(\frac{-D}{q}\right)=\prod_{r=1}^{q-1}\left(c \sin \frac{\pi}{q} r+b \sqrt{D} \cos \frac{\pi}{q} r\right) .
$$

Hence we get because $\sin \varphi=\sin (\pi-\varphi), \cos \varphi=\cdots \cos (\pi-\varphi)$ and $b^{2}=1$ 


$$
\left(\frac{-D}{q}\right)=\prod_{r=1}^{\frac{1}{1}(q-1)}\left(c^{2} \sin ^{2} \frac{\pi}{q} r-D \cos ^{2} \frac{\pi}{q} r\right)
$$

From (6) we get

If

$$
\left(\frac{-D}{q}\right)=\prod_{r=1}^{\frac{1}{2}(q-1)}\left(4 y \sin ^{2} \frac{\pi}{q} r-D\right)
$$

$$
4 y \sin ^{2} \frac{\pi}{q}-D \geqq 4 \sin ^{2} \frac{\pi}{q}
$$

we have, since $\sin x$ is increasing in the interval $0<x<\frac{\pi}{2}$

$$
\prod_{r=1}^{\frac{r}{(q-1)}}\left(4 y \sin ^{2} \frac{\pi}{q} r-D\right) \geqq \prod_{r=1}^{\frac{1}{2}(q-1)}\left(2 \sin \frac{\pi}{q} r\right)^{2}=q>1 .
$$

Hence we must have

$$
4 y \sin ^{2} \frac{\pi}{q}-D<4 \sin ^{2} \frac{\pi}{q}
$$

i. e.

$$
y<\frac{1}{4} D \operatorname{cosec}^{2} \frac{\pi}{q}+1
$$

and we get the following result:

Theorem 3. When $h(\sqrt{-D})$ is not divisible by $q$, the integral solutions $y$ of the equation (1) are all less than the number

$$
\frac{1}{4} D \operatorname{cosec}^{2} \frac{\pi}{q}+1
$$

$\S 5$.

We shall prove the following proposition:

Theorem 4. The equation

$$
x^{2}+x+\frac{1}{4}(q+1)=y^{q}
$$

is unsolvable in integers $x$ and $y$, if $q>3$ is a prime $\equiv 3$ (mod. 4).

In an imaginary quadratic field $K(\sqrt{d})$ with the discriminant $d(d<-4)$ the number of ideal classes $h(\sqrt{d})$ is given by the formula ${ }^{5}$

$$
h(\sqrt{d})=-\frac{1}{|d|} \sum_{n=1}^{|d|-1} n \cdot\left(\frac{d}{n}\right)
$$




\section{B. PERSSON, On a diophantine equation in two unknowns}

where the characters $\left(\frac{d}{n}\right)$ can only have the values $0, \pm 1$. From this formula we get the following inequality

$$
h(\sqrt{d})<\frac{1}{|d|} \sum_{n=1}^{|d|-1} n=\frac{|d|-1}{2}<|d| .
$$

In the present case we have $d=-q$ and hence $h(\overline{-q})$ is not divisible by $q$. As was shown in $\S 3$ this involves $\mathfrak{a} \sim(1)$ in the equation (2). But the equation (5) is impossible, when $D=q$, since every term in the right member is divisible by $q$, while the left member is not divisible by $q$.

\section{$\S 6$.}

We next consider the special case $q=3$ supposing that $h(\sqrt{-D})$ is not divisible by 3 . From (5) and (6) we then get

and

$$
4\left(\frac{-D}{3}\right)=3 c^{2}-D
$$

$$
y=\frac{1}{4}\left(D+c^{2}\right)=\frac{1}{3}\left(D-\left(\frac{D}{3}\right)\right) .
$$

We get the following result:

Theorem 5. If $D$ is positive integer $\equiv 3$ (mod. 4) having no squared factor $>1$ and if $h(\overline{-D})$ is not divisible by 3 , the equation

$$
x^{2}+x+\frac{1}{4}(D+1)=y^{3}
$$

has the only solution $y=\frac{1}{3}(D-1)$ if $D$ is of the form $3 c^{2}+4$, and the only solution $y=\frac{1}{3}(D+1)$ if $D$ is of the form $3 c^{2}-4$, and has no integral solutions for other values of $D$.

Remark. There are infinitely many integers without squared factor $>1$ of the form $3 c^{2}+4$ and $3 c^{2}-4 .^{6}$

Let us now suppose that the class number $h(\sqrt{-D})$ is divisible by 3 . In this case the equation

$$
x^{2}+x+\frac{1}{4}(D+1)=y^{3}
$$

can have other solutions than those given in the theorem 5. As examples we treat the cases $D=23,31,59,83$ where the class number has the value 3 .

I. $D=23$. The ideal classes in $K(\sqrt{-23})$ can be represented by the ideals (1), $(2, \varrho)$ and $\left(2, \varrho^{\prime}\right)$ where $\varrho=\frac{1}{2}(-1+\sqrt{-23})$. 
We have

The equation

$$
(2, \varrho) \cdot\left(2, \varrho^{\prime}\right)=(2) \quad \text { and } \quad\left(2, \varrho^{\prime}\right)^{3}=(1-\varrho)
$$

$$
x^{2}+x+6=y^{3}
$$

gives as in the general case

$$
(x-\varrho)=\mathfrak{a}^{3}
$$

where $\mathfrak{a}$ is an ideal.

If $\mathfrak{a} \sim(1)$ we get as in theorem $5 y=\frac{1}{3}(23+1)=8$, since $23=3 \cdot 3^{2}-4$. If $a \sim(2, \varrho)$ we get from $(8)$

$$
\begin{aligned}
& \left(2, \varrho^{\prime}\right)^{3}(x-\varrho)=\left(2, \varrho^{\prime}\right)^{3} \mathfrak{a}^{3} \\
& (1-\varrho)(x-\varrho)=(a+b \varrho)^{3}
\end{aligned}
$$

where $a$ and $b$ are integers.

$$
x-6-\varrho(x+2)=a^{3}-18 a b^{2}+6 b^{3}+\varrho\left(3 a^{2} b-3 a b^{2}-5 b^{3}\right) .
$$

Hence we get the system

$$
\begin{gathered}
x-6=a^{3}-18 a b^{2}+6 b^{3} \\
-x-2=3 a^{2} b-3 a b^{2}-5 b^{3} .
\end{gathered}
$$

Hence after elimination of $x$

From (9) we get

$$
-8=a^{3}+3 a^{2} b-21 a b^{2}+b^{3}
$$

$$
2 y=N(a+b \varrho)=a^{2}-a b+6 b^{2} .
$$

The equation (10) has the solutions

$$
\left\{\begin{array}{l}
a=-2 \\
b=0
\end{array} ; \quad\left\{\begin{array} { l } 
{ a = 3 } \\
{ b = 1 }
\end{array} \text { and } \quad \left\{\begin{array}{l}
a=0 \\
b=-2
\end{array}\right.\right.\right.
$$

which give the solutions $y=2, y=6$ and $y=12$ respectively of (7).

The case $\mathfrak{a} \sim\left(2, \varrho^{\prime}\right)$ leads to $(10)$ too. We see that by replacing $x$ by $-1-x, a$ by $-a$ and $b$ by $-b$ in (8) and (9).

II. $D=31$. As representatives of the ideal classes we choose $(1),(2, \varrho)$ and $\left(2, \varrho^{\prime}\right)$ with $\varrho=\frac{1}{2}(-1+\sqrt{-31})$.

We have

The equation

$$
(2, \varrho) \cdot\left(2, \varrho^{\prime}\right)=(2) \quad \text { and } \quad\left(2, \varrho^{\prime}\right)^{3}=\left(\varrho^{\prime}\right)
$$

$$
x^{2}+x+8=y^{3}
$$

gives

$$
(x-\varrho)=a^{3} .
$$


B. PERSSON, On a diophantine equation in two unknowns

If $\mathfrak{a} \sim(1)$ we get as in theorem $5 y=\frac{1}{3}(31-1)=10$, since $31=3 \cdot 3^{2}+4$. If $a \sim(2, \varrho)$ we get from $(12)$

$$
\varrho^{\prime}(x-\varrho)=(a+b \varrho)^{3}
$$

where $a$ and $b$ are integers.

$$
-x-8-\varrho x=a^{3}-24 a b^{2}+8 b^{3}+\varrho\left(3 a^{2} b-3 a b^{2}-7 b^{3}\right) .
$$

Hence

with

$$
-8=a^{3}-3 a^{2} b-21 a b^{2}+15 b^{3}
$$

$$
2 y=a^{2}-a b+8 b^{2} \text {. }
$$

The equation (13) has the solutions

$$
\left\{\begin{array}{l}
a=-2 \\
b=0
\end{array} ;\left\{\begin{array} { l } 
{ a = 1 } \\
{ b = 1 }
\end{array} \text { and } \left\{\begin{array}{l}
a=4 \\
b=6
\end{array}\right.\right.\right.
$$

which give the solutions $y=2, y=4$ and $y=140$ respectively of (11). $\mathfrak{a} \sim\left(2, \varrho^{\prime}\right)$ gives the same solutions.

III. $D=59$. As representatives of the ideal classes we choose $(1),(3, \varrho)$ and $\left(3, \varrho^{\prime}\right)$ with $\varrho=\frac{1}{2}(-1+\sqrt{-59})$.

We have

The equation

$$
(3, \varrho) \cdot\left(3, \varrho^{\prime}\right)=(3) \quad \text { and } \quad\left(3, \varrho^{\prime}\right)^{3}=\left(\varrho^{\prime}-3\right)
$$

gives

$$
x^{2}+x+15=y^{3}
$$

$$
(x-\varrho)=\mathfrak{a}^{3} .
$$

$\mathfrak{a} \sim(1)$ gives no solution of (14).

If $\mathfrak{a} \sim(3, \varrho)$ we get from (15)

$$
\begin{gathered}
\left(\varrho^{\prime}-3\right)(x-\varrho)=(a+b \varrho)^{3} \\
-4 x-15+\varrho(3-x)=a^{3}-45 a b^{2}+15 b^{3}+\varrho\left(3 a^{2} b-3 a b^{2}-14 b^{3}\right)
\end{gathered}
$$

Hence

with

$$
-27=a^{3}-12 a^{2} b-33 a b^{2}+71 b^{3}
$$

$$
3 y=a^{2}-a b+15 b^{2} \text {. }
$$

The equation (16) has the solutions

$$
\left\{\begin{array} { l } 
{ a = - 3 } \\
{ b = 0 }
\end{array} \quad \text { and } \quad \left\{\begin{array}{l}
a=-1 \\
b=-1
\end{array}\right.\right.
$$

which give the solutions $y=3$ and $y=5$ respectively of (14). 
IV. $D=83$. As representatives of the ideal classes we choose $(1),(3, \varrho)$ and $\left(3, \varrho^{\prime}\right)$ with $\varrho=\frac{1}{2}(-1+\sqrt{-83})$.

We have

The equation

$$
(3, \varrho) \cdot\left(3, \varrho^{\prime}\right)=(3) \text { and }\left(3, \varrho^{\prime}\right)^{3}=\left(\varrho^{\prime}+3\right)
$$

gives

$$
x^{2}+x+21=y^{3}
$$

$$
(x-\varrho)=a^{3} .
$$

$\mathfrak{a} \sim(1)$ gives no solution of (17).

If $\mathfrak{a} \sim(3, \varrho)$ we get from (18)

with

$$
\left(\varrho^{\prime}+3\right)(x-\varrho)=(a+b \varrho)^{3}
$$

$$
3 y=a^{2}-a b+21 b^{2} \text {. }
$$

$2 x-21+\varrho(-x-3)=a^{3}-63 a b^{2}+21 b^{3}+\varrho\left(3 a^{2} b-3 a b^{2}-20 b^{3}\right)$.

Hence

$$
-27=a^{3}+6 a^{2} b-69 a b^{2}-19 b^{3} .
$$

The equation (19) has the solution $a=-3, b=0$ which gives the solution $y=3$ of $(17)$.

At the end of this paper we give a table containing solutions of the equation

$$
x^{2}+x+\frac{1}{4}(D+1)=y^{3}
$$

when $D$ is a prime $<100$.

\section{$\S 7$.}

Let us examine the case $q=5$, when $h(\sqrt{-D})$ is not divisible by 5 . We get from (5)

$$
2^{4}\left(\frac{D}{5}\right)=5 c^{4}-10 c^{2} D+D^{2}
$$

Hence we find that the equation

$$
x^{2}+x+\frac{1}{4}(D+1)=y^{5}
$$

has at most one solution $y$. For if there were different values of $c$ for a given $D$, we get from (20)

and hence

$$
5 c_{1}^{4}-10 c_{1}^{2} D=5 c_{2}^{4}-10 c_{2}^{2} D
$$

i. e.

$$
c_{1}^{2}+c_{2}^{2}=2 D
$$

$$
2 D \equiv 2(\bmod .8)
$$

which is impossible since $D \equiv 3(\bmod .4)$. 
B. PERSSON, On a diophantine equation in two unknowns

If we put with an odd $z$

$$
D-5 c^{2}=2 z
$$

the equation (20) is transformed into

$$
4\left(\frac{D}{5}\right)=z^{2}-5 c^{4}
$$

I. If $\left(\frac{D}{5}\right)=1$ we get

$$
z^{2}-4=5 c^{4}
$$

$\left(22^{\prime}\right)$ has the only solution $z=3$ and $c==1$ in odd positive $z$ and $c$. This can be proved in the following way. $\left(22^{\prime}\right)$ can be written

with

$$
(z+2)(z-2)=5 c^{4}
$$

$$
(z+2, z-2)=1 \text {. }
$$

Hence we get the system $(z>0)$

$$
z \pm 2=5 a^{4} ; \quad z \mp 2=b^{4}
$$

where $(a, b)=1$ and $a b=c$.

Hence

$$
\pm 4=5 a^{4}-b^{4}
$$

where the lower sign is impossible, since the right member is congruent 4 modulo 16. Hence

$$
b^{4}+4=5 a^{4}
$$

which can be written

where

$$
\left(b^{2}+2 b+2\right)\left(b^{2}-2 b+2\right)=5 a^{4}
$$

$$
\left(b^{2}+2 b+2, b^{2}-2 b+2\right)=\left(b^{2}+2 b+2,4 b\right)=1 .
$$

Hence we get the system

$$
b^{2} \pm 2 b+2=5 f^{4} ; \quad b^{2} \mp 2 b+2=g^{4}
$$

with $(f, g)=1$ and $f g=a$.

The last equation can be written

$$
(b \mp 1)^{2}+1=g^{4}
$$

but as is well known the diophantine equation

$$
x^{4}-y^{4}=z^{2}
$$


has the only solution $z=0$. Hence $b= \pm 1$ is the only solution of the system. (A) and hence $z= \pm 3, c^{2}=1$ the only solutions of $\left(22^{\prime}\right)$ in odd integers.

This gives by $(21)^{-} D=11$ as the only case when the equation

$$
x^{2}+x+\frac{1}{4}(D+1) \cdots y^{5}
$$

is solvable if $\left(\frac{D}{5}\right)=\mathrm{i}$.

$$
15^{2}+15+1(11+1)=3^{5}
$$

II. If $\left(\begin{array}{l}D \\ 5\end{array}\right)=-1,(22)$ becomes

$$
z^{2}+4=5 c^{4}
$$

The equation $\left(22^{\prime \prime}\right)$ has only the solutions $z= \pm 1, c^{2}=1$ according to an information from $W$. LJUNGGREN not yet published.

This gives by (21) $D=7$ and $D=3$ as the only cases of solvability if $\left(\frac{D}{5}\right)=-1$.

$$
5^{2}+5+\frac{1}{4}(7+1)=2^{5}
$$

We get the following result:

Theorem 6. If $D$ is a positive integer $\equiv 3$ (mod. 4) having no squared factor $>1$ and if $h(\sqrt{-D})$ is not divisible by 5 , the equation

$$
x^{2}+x+\frac{1}{4}(D+1)=y^{5}
$$

is solvable in integers $x$ and $y$ only when $D=3,7$ and 11. In these cases the equation has a single solution $y$.

$$
\S 8 .
$$

Finally we consider the special case $q=7$, when $h(\sqrt{-D})$ is not divisible by 7 . We shall show that the equation

$$
x^{2}+x+\frac{1}{4}(D+1)=y^{7}
$$

has at most one solution $y$ for a given $D$.

From (5) we get

$$
7 c^{6}-35 c^{4} D+21 c^{2} D^{2}-D^{3}+2^{6}\left(\frac{D}{7}\right)=0 .
$$

Hence we get from (6) for the solutions $y$ of the equation (23)

$$
7 y^{3}-14 D y^{2}+7 D^{2} y-D^{3}-\frac{D}{+}\left(\frac{D}{7}\right)=0 .
$$




\section{B. PERSSON, On a diophantine equation in two unknowns}

Let $y_{1}, y_{2}$ and $y_{3}$ be the roots of the equation (25). We have

$$
\begin{gathered}
y_{1}+y_{2}+y_{3}=2 D \\
y_{1} y_{2}+y_{2} y_{3}+y_{3} y_{1}=D^{2} \\
7 y_{1} y_{2} y_{3}=D^{3}-\left(\begin{array}{c}
D \\
7
\end{array}\right)
\end{gathered}
$$

Hence we see that the equation (23) cannot have three solutions $y$. For if $D \equiv-1$ (mod. 8) we would have by (23) $y_{i} \equiv 0(\bmod .2)$ for $i=1,2,3$ against (II). If $D \equiv 3(\bmod .8)$ we would have by (23) $y_{i} \equiv 1(\bmod .2)$ against (I). Neither can we have two solutions for $D \equiv-1(\bmod .8)$ according to (II). That it is the same in the case $D \equiv 3(\bmod .8)$ can be seen in the following way.

Let (25) have three integral roots $y_{1}, y_{2}, y_{3}$. We put $y_{1}+y_{2}=u ; y_{1} y_{2}=v$. Hence by (I) and (II)

$$
u+y_{3}=2 D ; \quad v+y_{3} u=D^{2}
$$

and after elimination of $y_{3}$

$$
y_{1} y_{2}=v=(D-u)^{2}
$$

The $y_{i}$ are relatively prime two and two by (II), since $\left(D, y_{i}\right)=1$ by (25). Hence all $y_{i}$ are squares according to (26). If $y_{1}$ and $y_{2}$ are odd we get $y_{3} \equiv 4(\bmod .8)$ by $(\mathrm{I})$ and $\left(\frac{D}{7}\right)=-1$ in (III). Hence the relation (III) can be written

$$
7 A^{2}=D^{3}+1
$$

where $A$ is an integer.

This equation has the only integral solutions $D=-1$ and $D=3$. $^{7}$ With $D=3$ we get $y_{1}=y_{2}=1$ and $y_{3}=4$.

If we put

$$
D=4 z+7 c^{2}
$$

the equation (24) is transformed into

$$
z^{3}-7 c^{4} z-7 c^{6}=\left(\frac{D}{7}\right)
$$

For $c=1$ we get, if $\left(\frac{D}{7}\right)=-1$, the solutions $z=3 ; z=-1$ and $z=-2$ of $\left(24^{\prime}\right)$. The two first values give $D=19$ and $D=3$ respectively. Hence the equation

$$
x^{2}+x+5=y^{7}
$$

has the only solution $y=5$.

$$
279^{2}+279+\frac{1}{4}(19+1)=5^{7} .
$$




\section{Table}

containing solutions of the equation

$$
x^{2}+x+\frac{1}{4}(D+1)=y^{3}
$$

when $D$ is a prime $<100$.

\begin{tabular}{|c|c|c|c|c|c|c|c|}
\hline \multirow{3}{*}{$D$} & \multirow{3}{*}{$h(\boldsymbol{V}-D)$} & \multicolumn{2}{|c|}{$D=$} & \multirow{2}{*}{\multicolumn{2}{|c|}{$\begin{array}{l}\text { Solution when } \\
\qquad a \sim(1)\end{array}$}} & \multirow{2}{*}{\multicolumn{2}{|c|}{ Other solutions }} \\
\hline & & $3 c^{2}+4$ & $3 c^{2}-4$ & & & & \\
\hline & & $c$ & $c$ & $y$ & $x(>0)$ & $y$ & $x(\geqq 0)$ \\
\hline 7 & 1 & 1 & $\cdots$ & 2 & 2 & $\ldots$ & - \\
\hline 11 & 1 & - & - & - & - & - & - \\
\hline 19 & 1 & - & - & - & - & - & 一 \\
\hline 23 & 3 & $一$ & 3 & 8 & 22 & $\begin{array}{r}2 \\
6 \\
12\end{array}$ & $\begin{array}{r}1 \\
14 \\
41\end{array}$ \\
\hline 31 & 3 & 3 & - & 10 & $3 \mathrm{l}$ & $\begin{array}{r}2 \\
4 \\
140\end{array}$ & $\begin{array}{r}0 \\
7 \\
1656\end{array}$ \\
\hline 43 & 1 & - & - & - & - & - & 一 \\
\hline 47 & 5 & - & - & - & - & - & $\longrightarrow$ \\
\hline 59 & 3 & - & - & - & - & $\begin{array}{l}3 \\
\mathbf{5}\end{array}$ & $\begin{array}{r}3 \\
10\end{array}$ \\
\hline 67 & 1 & 一 & - & - & - & - & - \\
\hline 71 & 7 & - & 5 & 24 & 117 & - & $\longrightarrow$ \\
\hline 79 & 5 & 5 & - & 26 & 132 & - & - \\
\hline 83 & 3 & - & -- & - & - & 3 & 2 \\
\hline
\end{tabular}

LITERATURE. 1. T. Nagell: :Des équations indéterminées $x^{2}+x+1=y^{n}$ et $x^{2}+x+1=3 y^{n_{*}}$, Norsk matematisk forenings skrifter. Serie $\mathrm{I}, \mathrm{Nr}$ 2, Oslo, 1921. - 2. W. Ljunggren: „Einige Bemerkungen über die Darstelıung ganzer Zahlen durch binäre kubische Formen mit positiver Diskriminante», Acta math. Vol. 75, 1942. - 3. A. Thue: Utber die Unlösbarkeit der Gleichung $a x^{2}+b x+c=d y^{n}$ in grossen ganzen Zahlen $x$ und $y^{\prime \prime}$, Archiv for Mathematik og Naturvidenskab. B. XXXIV, Kristiania, 1916. - 4. - - "Über Annäherungswerte algebraischer Zahlen", Journal für Math., Band 135 (1909). - 5. E. Hecke: "Vorlesungen über die Theorie der algebraischen Zahlen». Leipzig 1923. \$ 52, Satz 152; formula 1. 6. T. Nagell: "Zur Arithmetik der Polynome", Abh. Math. Sem. Univ. Hamburg. I. Band, 1922. - 7. W. Ljunggren: "Einige Sätze über umbestimmte Gleichungen von der Form $A x^{4}+B x^{2}-C=D y^{2}$. Vidensk. Akad. Oslo. I Mat. Naturv. K1. 1942. No 9.

Tryckt den 1 april 1949

Uppsala 1949. Almqvist \& Wiksells Boktryckeri AB 